\title{
The water relations of growth and polyhydroxy alcohol production by ascomycetous yeasts
}

\author{
J. H. VAN EcK, ${ }^{1}$ B. A. Prior ${ }^{1 *}$ and E. V. BrandT ${ }^{2}$ \\ Department of Microbiology and Biochemistry, and Department of Chemistry ${ }^{2}$, University of the Orange Free State, \\ PO Box 339, Bloemfontein, 9300 South Africa
}

(Received 30 June 1992; revised 31 December 1992; accepted 7 January 1993)

\begin{abstract}
The response of 31 ascomycetous yeasts to a reduction in water activity $\left(a_{\mathrm{w}}\right)$ adjusted with D-glucose or $\mathrm{NaCl}$ was investigated. The growth of most yeasts was more tolerant to glucose than to $\mathrm{NaCl}$ at equivalent $a_{\mathrm{w}}$. Zygosaccharomyces rouxii was the most osmotolerant yeast examined. Natural abundance ${ }^{13} \mathrm{C}-\mathrm{NMR}$ spectroscopy and HPLC analyses of eight yeasts indicated that glycerol and arabitol or mannitol were accumulated intracellularly in response to $a_{\mathrm{w}}$ reduction. Pichia sorbitophila, Candida cacaoi, Candida magnoliae and $Z y$ gosaccharomyces bisporus responded to reduced $a_{\mathrm{w}}$ by a decrease in specific growth rate and cell volume, and an accumulation of glycerol. The other polyol accumulated did not increase in concentration with $a_{\mathrm{w}}$ reduction to the same degree as glycerol. A polyol concentration ratio (intra/extracellular) as high as 800-fold was attained across the membrane. Greater amounts of polyols were produced at equivalent $a_{w}$ values when adjusted with glucose than with $\mathrm{NaCl}$. The ability to accumulate high concentrations of polyols appears to be the most important criterion in determining osmotolerance.
\end{abstract}

\section{Introduction}

Low molecular mass compounds are accumulated intracellularly in most organisms when exposed to osmotic stress to equilibrate the cytoplasmic water activity $\left(a_{\mathrm{w}}\right)$ with the $a_{\mathrm{w}}$ of the surrounding environment (Yancey et al., 1982; Csonka, 1989). The main solutes accumulated in yeasts exposed to osmotic stress are polyhydroxy alcohols (polyols) such as glycerol, D-arabitol, Dmannitol and meso-erythritol (Spencer \& Spencer, 1978), and are compatible with metabolic activity (known as compatible solutes; Brown, 1978). The polyol accumulated is related to the yeast species, the growth phase of the yeast (Nobre \& da Costa, 1985) and the carbon source used for growth (Van Eck et al., 1989). The terms 'osmophilic' and 'osmotolerant' have been widely used to describe the water relations of yeasts. Whereas relatively few yeasts can be described as having a requirement for reduced $a_{\mathrm{w}}$ (i.e. osmophilic; Jermini \& Schmidt-Lorenz, 1987), many yeasts are described in the taxonomic literature as osmotolerant by virtue of their ability to grow in the presence of $60 \%$ glucose (Barnett et al., 1983). The distinction between osmotolerant and non-osmotolerant yeasts, however, is poorly understood on a genetic and physiological basis, and the relationship

\footnotetext{
*Author for correspondence. Tel. 51401 2396; fax 51482004.
}

of polyol accumulation to osmotolerance has only been described in a few yeasts (Adler et al., 1985; Edgley \& Brown, 1978; Nobre \& da Costa, 1985; Reed et al., 1987; Van Eck et al., 1989). The purpose of this study was to investigate the water relations of a wide range of yeasts in terms of their growth requirements, cell volume, types of solutes accumulated and the characteristics of the accumulation process in a selected group of yeasts. Most of the yeasts investigated were chosen on the basis of their frequent occurrence in high-sugar and high-salt foods (Barnett et al., 1983).

\section{Methods}

Organisms and media. Yeasts were obtained from J. P. van der Walt of the Council for Scientific and Industrial Research (CSIR), Pretoria, South Africa, the Centraalbureau voor Schimmelcultures (CBS), Delft, the Netherlands, C. P. Kurtzman, Northern Regional Research Laboratory (NRRL), Peoria, Illinois, USA and the American Type Culture Collection (ATCC), Rockville, Maryland, USA (details in legend to Fig. 1). The cultures were maintained on YM agar slants (Wickerham, 1951). All growth experiments were conducted in yeast nitrogen base

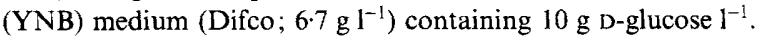

Growth studies. The inoculum for each yeast was prepared by seeding glucose-YNB medium $(100 \mathrm{ml})$ in $500 \mathrm{ml}$ Erlenmeyer flasks and culturing with vigorous agitation on a rotary shaker (180 r.p.m.) at $30^{\circ} \mathrm{C}$ until late exponential growth phase. The inoculum $(0.5 \mathrm{ml})$ was used to seed glucose-YNB medium $(10 \mathrm{ml})$ in tubes. The $a_{\mathrm{w}}$ of the medium was adjusted with either D-glucose (Norrish, 1966) or $\mathrm{NaCl}$ (Robinson \& Stokes, 1959). The tubes were incubated at $30^{\circ} \mathrm{C}$ with 


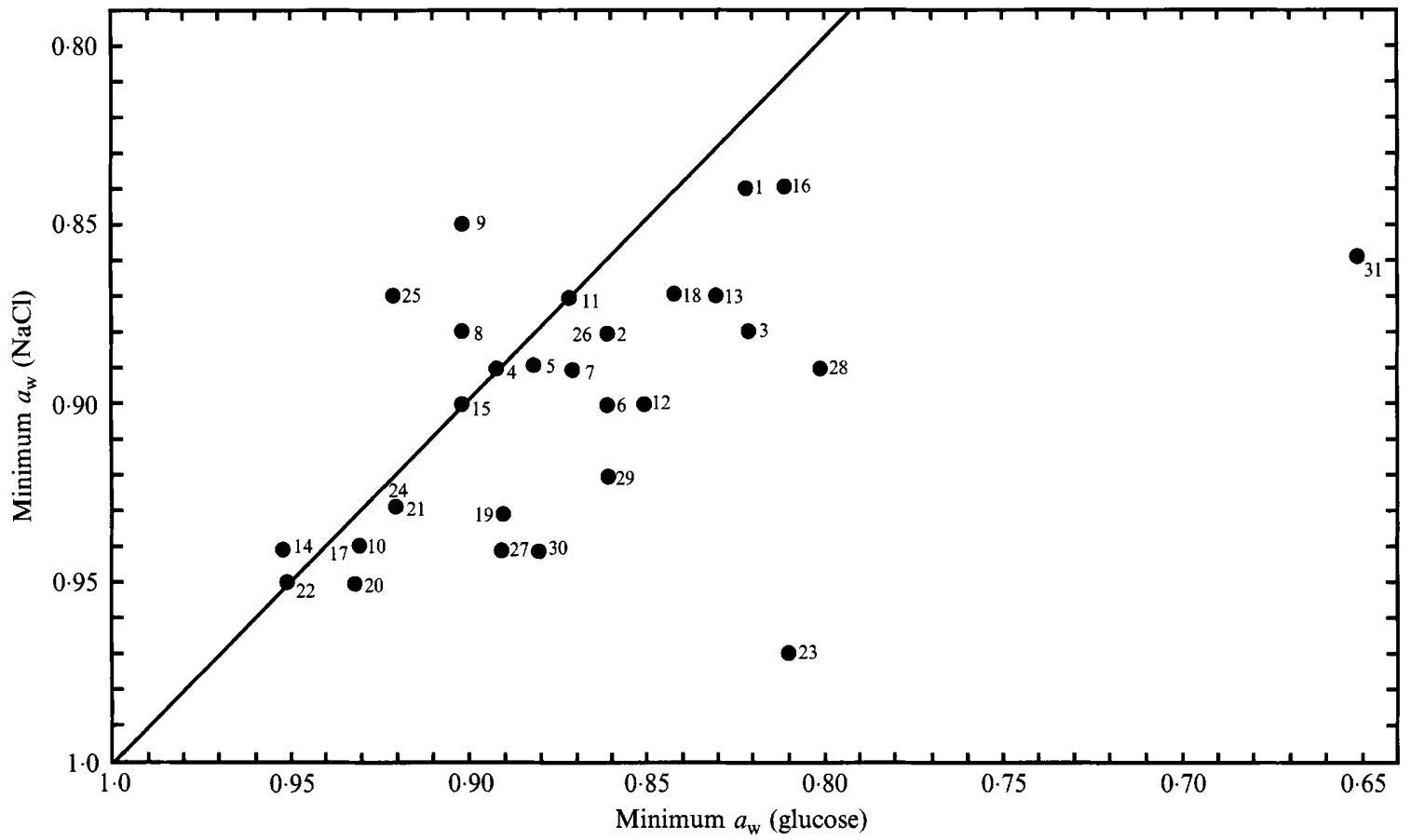

Fig. 1. The minimum $a_{\mathrm{w}}(\mathrm{NaCl}$ or D-glucose) as determined in tubes for the growth of the following ascomycetous yeasts in glucoseYNB medium: 1, Candida cacaoi CBS 2020; 2, Candida homilentoma CSIR Y917; 3, Candida magnoliae NRRL YB4226; 4, Candida silvicultrix CSIR Y481; 5, Candida tropicalis CBS 94; 6, Citromyces matritensis CSIR Y159; 7, Debaryomyces castellii CSIR Y229; 8, Debaryomyces hansenii CSIR Y953; 9, Geotrichium terrestre CSIR Y803; 10, Hansenula anomala CSIR Y207; 11, Hansenula ciferrii CSIR Y804; 12, Hansenula jadinii CSIR Y227; 13, Hansenula sydowiorum CSIR Y463; 14, Lipomyces kononenkoae CSIR Y353; 15, Pichia farinosa CSIR Y226; 16, Pichia membraneaefaciens CSIR Y35; 17, Pichia sorbitophila CSIR Y170; 18, Pichia stipitis CSIR Y567; 19, Saccharomyces cerevisiae ATCC 4126; 20, Saccharomyces dairensis CSIR Y844; 21, Saccharomyces exiguus CSIR Y847; 22, Schizosaccharomyces octosporus CSIR Y934; 23, Schizosaccharomyces pombe CSIR Y457; 24, Schwanniomyces occidentalis CSIR Y936; 25, Stephanoascus ciferrii CSIR Y487; 26, Wingea robertsiae CSIR Y446; 27, Zygosaccharomyces bailii CSIR Y126; 28, Zygosaccharomyces bisporus CSIR Y849; 29, Zygosaccharomyces fermentii CSIR Y860; 30, Zygosaccharomyces florentinus CSIR Y 576; 31, Zygosaccharomyces rouxii CSIR Y364. The diagonal line represents the division between yeasts with greater $\mathrm{NaCl}$ tolerance (upper left) and those with greater glucose tolerance (lower right).

weekly agitation on a Vortex mixer. Growth was monitored at $640 \mathrm{~nm}$ with a Klett-Summerson colorimeter. Three doublings in absorbance were taken to represent growth at the different $a_{\mathrm{w}}$ values.

Growth of and polyol accumulation (as identified by ${ }^{13} \mathrm{C}-\mathrm{NMR}$ spectroscopy and quantified by HPLC) by yeasts at reduced $a_{\mathrm{w}}$ values was examined by preparing inocula in $50 \mathrm{ml}$ glucose-YNB medium ( $250 \mathrm{ml}$ Erlenmeyer flasks) as described above. These cultures $(6.5 \mathrm{ml})$ were used to inoculate glucose-YNB $(130 \mathrm{ml})$ in $500 \mathrm{ml}$ Erlenmeyer flasks equipped with side-arm cuvettes. The cultures were incubated under the same conditions as the inocula. Growth was monitored with a Klett-Summerson colorimeter at $640 \mathrm{~nm}$ and the contents of the flasks were harvested for analyses by centrifugation $(15000 \mathrm{~g}$ for $10 \mathrm{~min}$ ) during the late exponential growth phase.

Analytical methods. Methods for the determination of biomass, cell volume, polyol concentration, calculation of the intracellular $a_{\mathrm{w}}$ and the identification of accumulated solutes by ${ }^{13} \mathrm{C}-\mathrm{NMR}$ spectroscopy were described previously (Van Eck et al., 1989).

\section{Results}

Minimum $a_{\mathrm{w}}$ for growth

The minimum $a_{\mathrm{w}}$ values for growth of the yeasts are shown in Fig. 1. Of the 31 yeasts evaluated, 23 tolerated glucose better than $\mathrm{NaCl}$ at equivalent $a_{\mathrm{w}}$ values, whereas four yeasts were more $\mathrm{NaCl}$-tolerant and four were equitolerant. Genera such as Pichia, Saccharomyces, Schizosaccharomyces and $Z$ ygosaccharomyces were more glucose-tolerant than $\mathrm{NaCl}$-tolerant at equivalent $a_{\mathrm{w}}$ values. Members of the genera Zygosaccharomyces, Pichia, Candida, Hansenula and Geotrichium were the most tolerant of the 13 genera evaluated. Zygosaccharomyces rouxii was exceptionally more osmotolerant than the other yeasts.

Identification of solutes accumulated by yeasts grown at 0.998 and $0.95 a_{\mathrm{w}}$ values (glucose or $\mathrm{NaCl}$ )

The ${ }^{13} \mathrm{C}-\mathrm{NMR}$ spectra of Candida magnoliae, Pichia sorbitophila and Saccharomyces cerevisiae grown at $0.998 a_{\mathrm{w}}$ indicated that mannitol and glycerol, arabitol and glycerol, and glycerol, respectively, were the main polyols accumulated (Fig. 2) with a large number of other compounds also present in significant concentrations (scans between 0 and 60, and 80 and 200 p.p.m. 


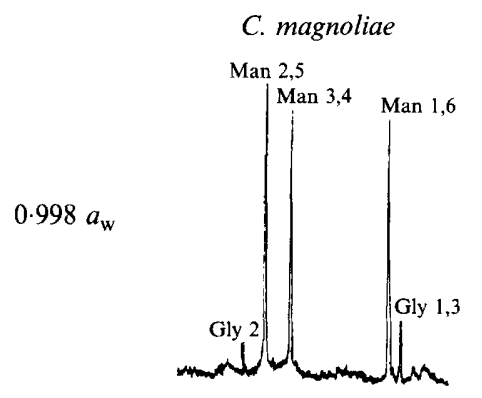

$$
\text { P. sorbitophila }
$$

Sacc. cerevisiae
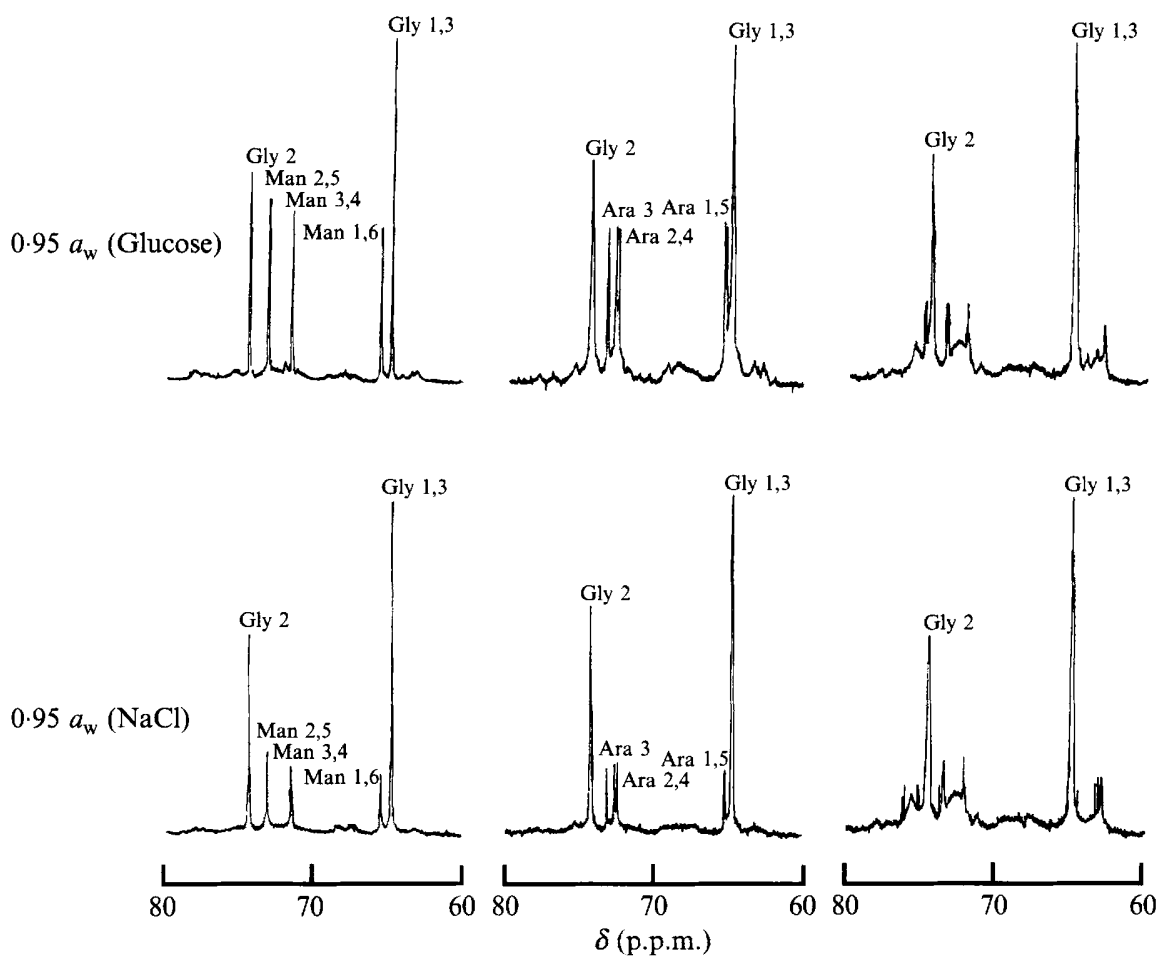

Fig. 2. Natural abundance ${ }^{13} \mathrm{C}$-NMR scans of C. magnoliae, P. sorbitophila and Sacc. cerevisiae washed cells grown at 0.998 and $0.95 a_{\mathrm{w}}$ ( $\mathrm{NaCl}$ or D-glucose) in glucose-YNB medium in shake flasks. Ara, arabitol; Gly, glycerol; Man, mannitol.

not shown). When the $a_{\mathrm{w}}$ of the medium was reduced to 0.95 with glucose or $\mathrm{NaCl}$, the peaks representing glycerol in all three yeasts increased in significance relative to the other peaks. However, the intensities of the spectra of each yeast are not comparable because of differences in gain. The peaks representing mannitol and arabitol found respectively in $C$. magnoliae and $P$. sorbitophila are apparently not regulated by osmotic stress. Sacc. cerevisiae under osmotic stress failed to accumulate another polyol. No other significant peaks were observed in scans between 0 and 200 p.p.m. (data not shown, except between 60 and 80 p.p.m.). ${ }^{13} \mathrm{C}$-NMR scans of cultures of Candida cacaoi, Candida silvicultrix, Debaryomyces hansenii, Zygosaccharomyces bisporus and $Z$. rouxii grown at $0.998 a_{\mathrm{w}}$ and at $0.95 a_{\mathrm{w}}(\mathrm{NaCl}$ or glucose) revealed that the only compounds osmotically regulated were glycerol and arabitol (data not shown).
Specific growth rate and cell volume at reduced $a_{\mathrm{w}}$

When the $a_{\mathrm{w}}$ was reduced from 0.998 to 0.92 with either glucose or $\mathrm{NaCl}$, the specific growth rates of $C$. cacaoi (Fig. 3), C. magnoliae (Fig. 4), P. sorbitophila (Fig. 5) and $Z$. bisporus (Fig. 6) decreased, which suggests that these yeasts are osmotolerant rather than osmophilic. The cell volume of these yeasts was also smaller when grown at reduced $a_{\mathrm{w}}$ values (Figs 3-6), indicating that volume regulation is an important mechanism of adaption by yeasts to reduced $a_{\mathrm{w}}$.

\section{Polyol accumulation at reduced $a_{\mathrm{w}}$}

Reduction in the $a_{\mathrm{w}}$ of the growth medium with glucose or $\mathrm{NaCl}$ resulted in a marked increase in the intracellular glycerol concentration of the four yeasts (Figs 3-6) and at $0.92 a_{\mathrm{w}}$, the glycerol concentration varied between 150 


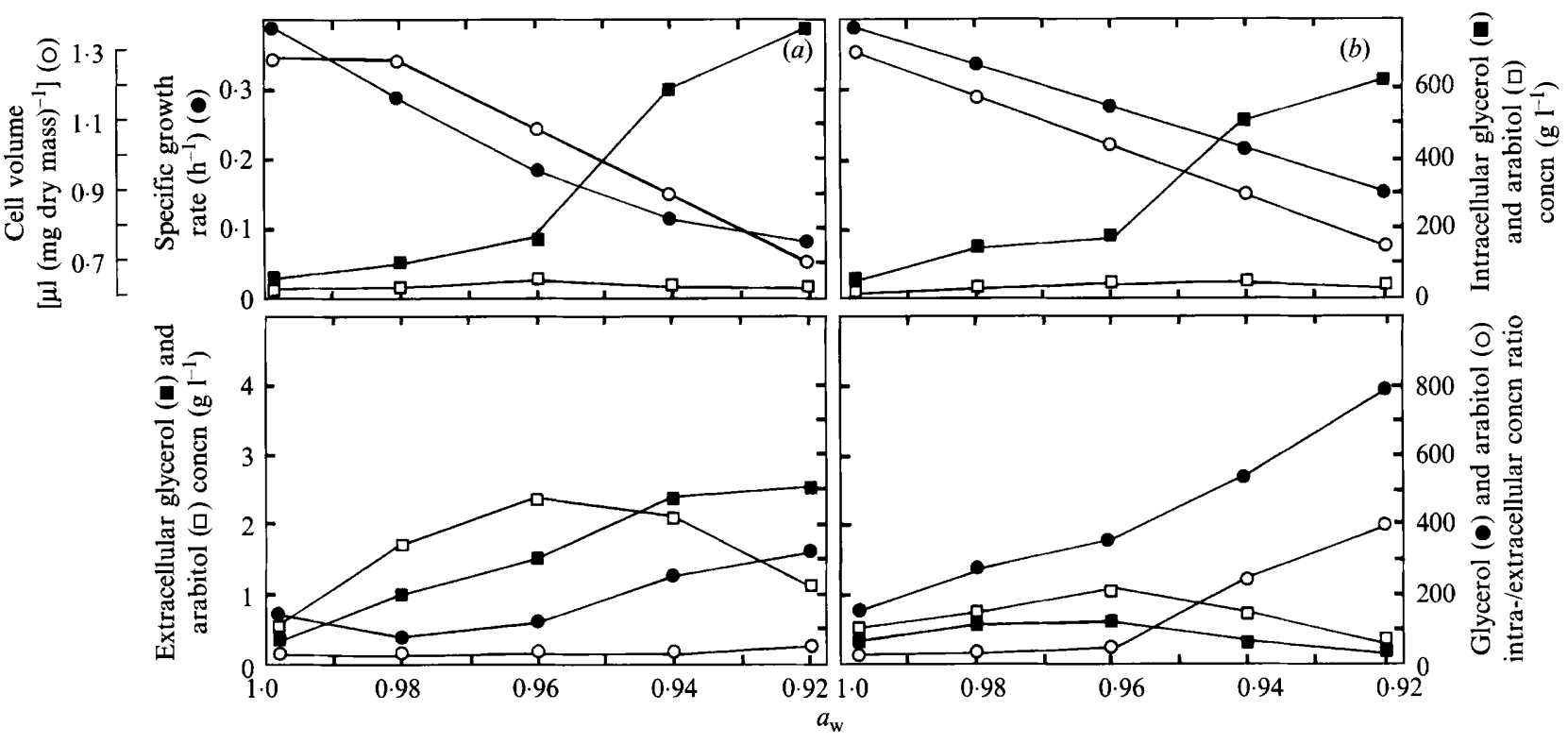

Fig. 3. Response of growth and polyol production by $C$. cacaoi in shake flasks containing glucose-YNB medium at $30^{\circ} \mathrm{C}$ to water reduction with D-glucose $(a)$ and $\mathrm{NaCl}(b)$.

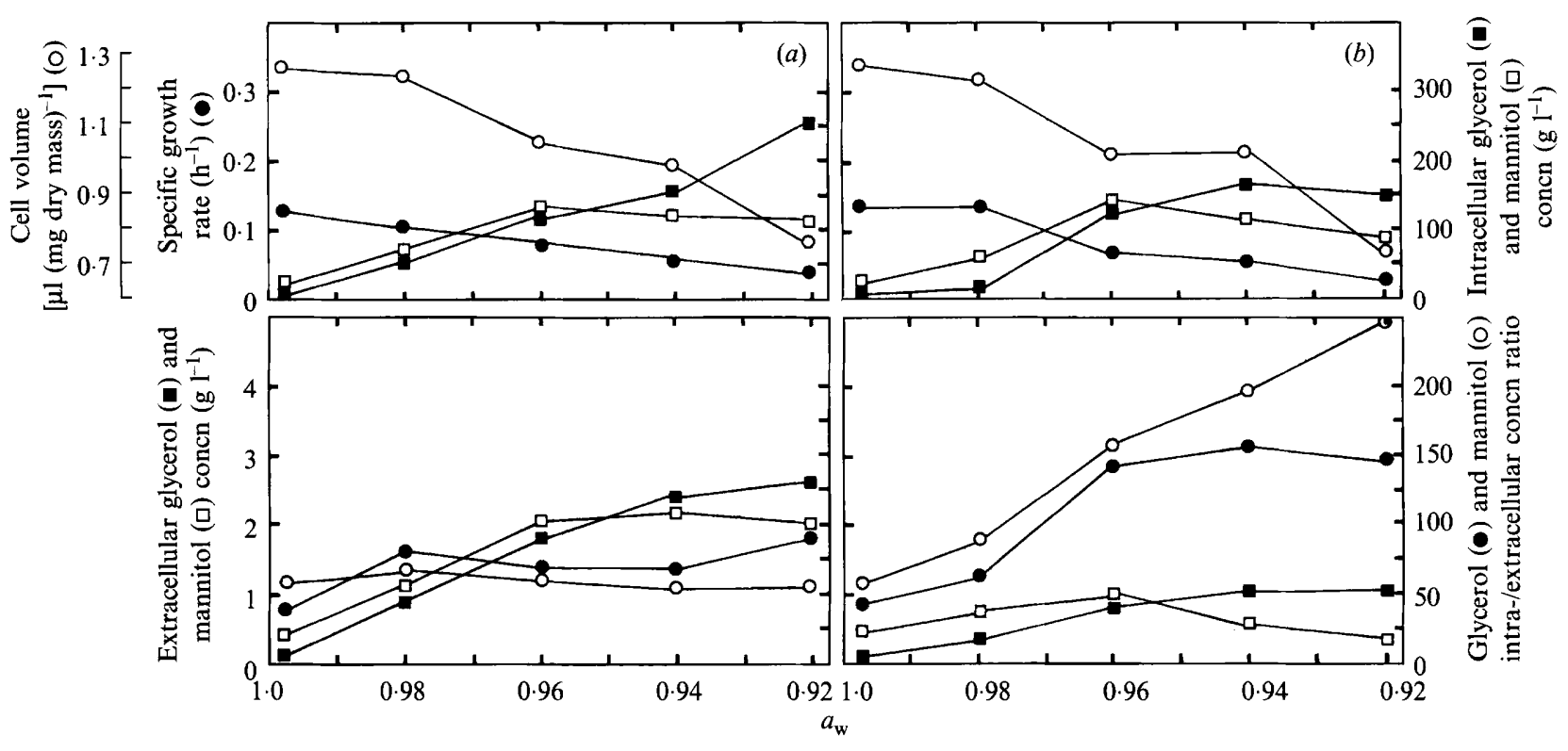

Fig. 4. Response of growth and polyol production by C. magnoliae in shake flasks containing glucose-YNB medium at $30^{\circ} \mathrm{C}$ to water reduction with D-glucose $(a)$ and $\mathrm{NaCl}(b)$.

and $760 \mathrm{~g}^{-1}$. The intracellular concentrations of arabitol (C. cacaoi, P. sorbitophila or Z. bisporus) or mannitol ( $C$. magnoliae) increased only slightly or not at all when the $a_{\mathrm{w}}$ of the growth medium was reduced, which suggests that these polyols are not as osmotically responsive as glycerol.

Extracellular polyol concentrations increased with a reduction of $a_{\mathrm{w}}$ (Figs 3-6) although in a number of instances, the concentrations decreased at $a_{\mathrm{w}}$ values less than 0.96 which suggests that a mechanism may operate to retard the leakage of the polyols from the cell. In contrast to marked differences in the intracellular concentrations of glycerol and the other polyols at reduced $a_{\mathrm{w}}$, the polyol concentrations in the medium 


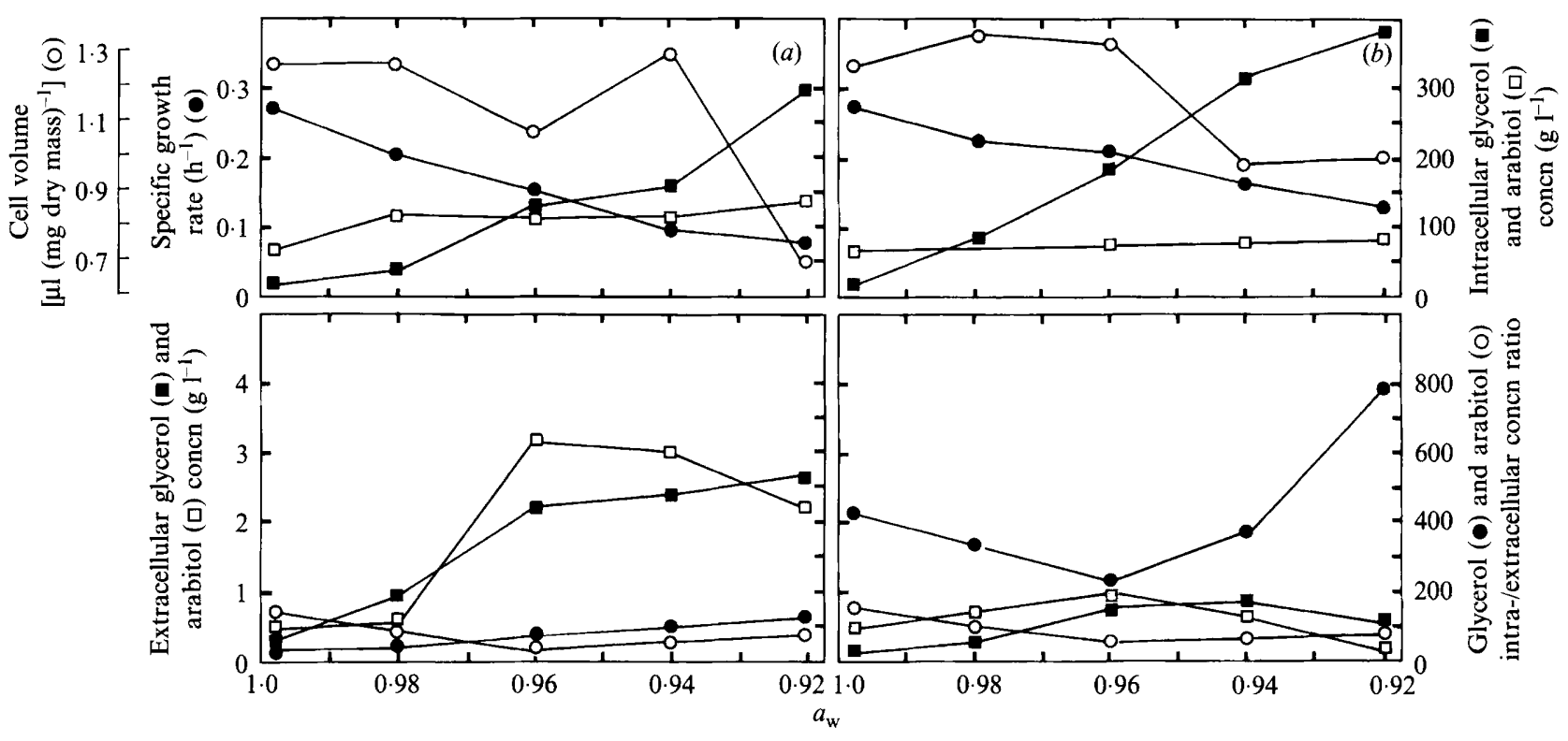

Fig. 5. Response of growth and polyol production by $P$. sorbitophila in shake flasks containing glucose-YNB medium at $30^{\circ} \mathrm{C}$ to water reduction with D-glucose $(a)$ and $\mathrm{NaCl}(b)$.

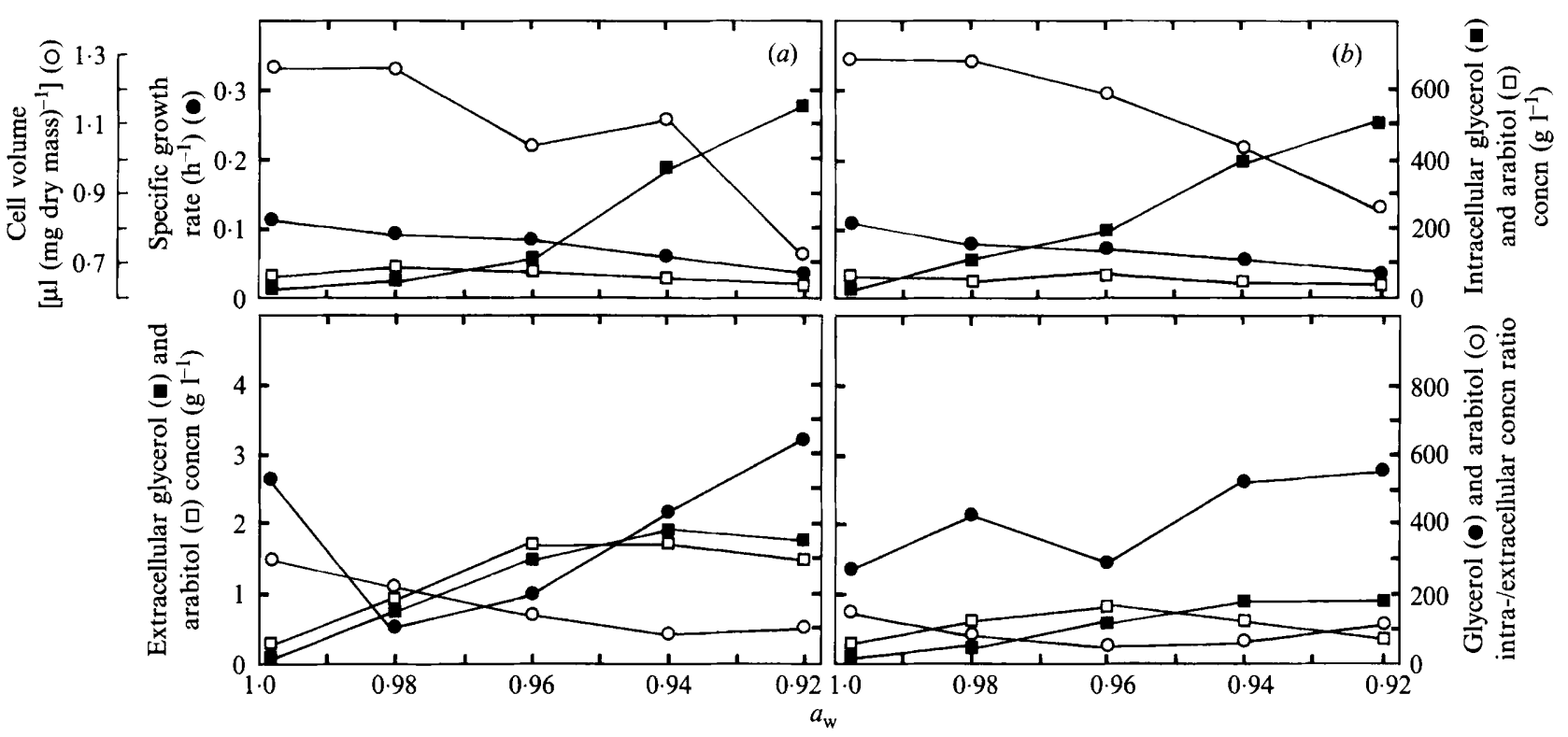

Fig. 6. Response of growth and polyol production by $Z$. bisporus in shake flasks containing glucose-YNB medium at $30^{\circ} \mathrm{C}$ to water reduction with D-glucose $(a)$ and $\mathrm{NaCl}(b)$.

were fairly similar. In general, the extracellular polyol concentrations were much greater when the $a_{\mathrm{w}}$ was adjusted with glucose than with $\mathrm{NaCl}$.

\section{Polyol concentration ratio (intra-/extracellular)}

In most instances, a reduction in the $a_{\mathrm{w}}$ to 0.92 resulted in an increase in the ratio of intracellular to extracellular glycerol concentrations (Figs 3-6) and at 0.92 $a_{\mathrm{w}}(\mathrm{NaCl}$ ), glycerol ratios as high as 800 were observed. In C. cacaoi (Fig. 3) and $P$. sorbitophila (Fig. 5) the glycerol ratios were greater in media adjusted with $\mathrm{NaCl}$ than with glucose. In $C$. magnoliae (Fig. 4) the reverse was observed, whereas in Z. bisporus (Fig. 6) no consistent pattern was found. The arabitol concentration ratio (or mannitol in C. magnoliae) increased only slightly or not at all, or in some instances decreased with a reduction of the $a_{\mathrm{w}}$. When the $a_{\mathrm{w}}$ was adjusted with $\mathrm{NaCl}$, the glycerol 


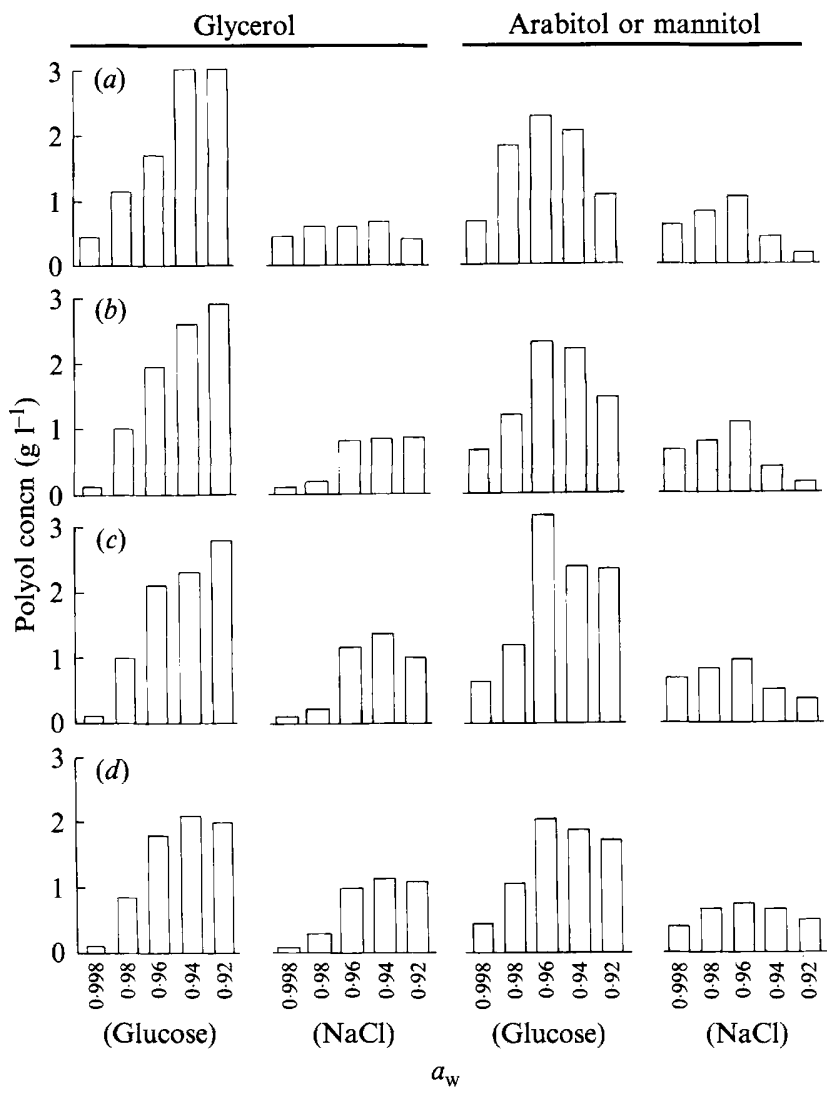

Fig. 7. Effect of $a_{\mathrm{w}}$ on the total production of polyols in shake flasks by (a) C. cacaoi (glycerol and arabitol), (b) C. magnoliae (glycerol and mannitol), (c) P. sorbitophila (glycerol and arabitol) and (d) Z. bisporus (glycerol and arabitol). The water activity of the glucose-YNB medium was adjusted with either D-glucose or $\mathrm{NaCl}$.

ratio was greater than the ratio of the other polyols in all instances except in C. magnoliae.

\section{Production of polyols}

The total concentration of glycerol produced (intra- and extracellular) increased with reduction of $a_{\mathrm{w}}$ and was highest at $0.92 a_{\mathrm{w}}$ (glucose) with the exception of $Z$. bisporus (Fig. $7 a$ ). In contrast, lower concentrations of glycerol were produced when the $a_{\mathrm{w}}$ was adjusted with $\mathrm{NaCl}$. Calculations, however, showed that the percentage glycerol retained within the cell was, in most instances, greater when the $a_{\mathrm{w}}$ was adjusted with $\mathrm{NaCl}$ than with glucose. In general, the percentage retained decreased with a reduction in $a_{\mathrm{w}}$. A notable exception was $C$. cacao $i$ which retained $70 \%$ of the total glycerol produced at $0.92 a_{\mathrm{w}}(\mathrm{NaCl})$.

Arabitol (or mannitol in C. magnoliae) production was greater in media adjusted with glucose than with $\mathrm{NaCl}$ and attained a maximum concentration at $0.96 a_{\mathrm{w}}$ (Fig. $7 b$ ). A marked reduction was observed at $a_{\mathrm{w}}$ values less than 0.96. Calculations of the percentage arabitol (or mannitol) retained intracellularly showed, in all instances, a decrease with $a_{\mathrm{w}}$.

\section{Discussion}

The minimum $a_{\mathrm{w}}$ values for the 31 yeasts reported here are, in general, similar to values disclosed elsewhere for a limited number of ascomycetous yeasts (Tilbury, 1980; Tokuoka \& Ishitani, 1991). The minimum $a_{\mathrm{w}}$ for the growth of $Z$. rouxii has frequently been reported. The minimum value of $0.65 a_{\mathrm{w}}$ (glucose) found here is similar to $0.62 a_{\mathrm{w}}$ (sucrose) and $0.65 a_{\mathrm{w}}$ (glucose) reported by Restaino et al. (1983) and Tilbury (1980), respectively. Others have found that the minimum growth $a_{\mathrm{w}}$ for $Z$. rouxii, adjusted with glucose, fructose or sucrose, was much higher and may reflect differences between strains, carbon sources or medium composition (Bellinger \& Larher, 1988; Jermini \& Schmidt-Lorenz, 1987; Tokuoka \& Ishitani, 1991). Comparison of the minimum growth $a_{\mathrm{w}}$ values (glucose or $\mathrm{NaCl}$ ) reported here for $D$. hansenii, Hansenula anomala, Pichia membraneafaciens, Sacc. cerevisiae, Schizosaccharomyces pombe, Candida tropicalis and $Z$. bisporus with values determined by Tokuoka \& Ishitani (1991) showed similar values in most cases with a few notable exceptions. The greater tolerance of some yeasts to glucose or $\mathrm{NaCl}$ can be related to their ecology. For example, salt-tolerant $D$. hansenii is found largely in marine environments and salt foods (Barnett $e t$ al., 1983). Sugar-tolerant yeasts such as Zygosaccharomyces bailii, $Z$. florentinus and $Z$. rouxii are commonly isolated from foods containing high sugar concentrations (Barnett et al., 1983). Interestingly, Tokuoka \& Ishitani (1991) found that the $Z$. rouxii strains isolated from high-salt foods tolerated $\mathrm{NaCl}$ to a greater extent than those isolated from high-sugar foods. In all instances, however, the minimum growth $a_{\mathrm{w}}$ of these strains was lower when adjusted with glucose than with $\mathrm{NaCl}$. The ability of some strains and species to tolerate higher $\mathrm{NaCl}$ concentrations could be due to the presence of a sodium extrusion pump (Hobot \& Jennings, 1981; Norkrans \& Kylin, 1969). Some yeasts evidently transport glycerol into their cells with sodium ions (Lucas et al., 1990; Van Zyl et al., 1990), yet may not possess an efficient mechanism to expel sodium ions.

${ }^{13} \mathrm{C}$-NMR spectroscopy revealed that only glycerol, arabitol and mannitol were solutes whose concentrations were significantly increased during osmotic stress in ascomycetous yeasts. Glycerol was the major osmotically active solute found in the eight yeast species studied here as well as a total of 18 species investigated by Brown (1978), Reed et al. (1987), Meikle et al. (1991) and Bellinger \& Lahrer (1988). Furthermore, glycerol is the principal osmolyte in some filamentous fungi, algae, insects, crustaceans and vertebrates which indicates that 
there is a selective advantage to organisms using such low molecular mass solutes (Yancey et al., 1982; Hocking \& Norton, 1983). As an osmolyte, glycerol offers a number of advantages: high glycerol concentrations confer a remarkable degree of protection to enzymes and macromolecular structure (Brown, 1978), and modification of proteins is unnecessary in order to function in concentrated intracellular solutions. Furthermore, the solubility and viscosity of glycerol are hardly affected by increasing concentrations of this polyol (Chirife et al., 1983, 1984).

With the exception of Sacc. cerevisiae, ascomycetous yeasts were found to contain the polyols arabitol or mannitol. Intracellular accumulation of these solutes, however, does not appear to respond to osmotic stress to the same degree as observed with glycerol and their role in osmoregulation is uncertain. They may act as reserve compatible solutes as exhaustion of the energy source during the late exponential growth phase leads to the possible consumption of glycerol by $D$. hansenii (Nobre $\&$ da Costa, 1985) and H. anomala (Van Eck et al., 1989). Sacc. cerevisiae, however, failed to produce a secondary polyol, yet the degree of osmotolerance of this yeast was not significantly less than other yeasts that produced two polyols. These secondary solutes, however, may be important in sustaining viability when a yeast is exposed to a sudden osmotic shock (Brown et al., 1986; Van Zyl \& Prior, 1990).

Accumulation of other solutes, such as amino acids and betaines, has often been observed in bacteria and plants in response to osmotic stress (Yancey et al., 1982; Csonka, 1989). We failed to observe these solutes in the ${ }^{13} \mathrm{C}-\mathrm{NMR}$ spectra of the yeasts investigated and it would appear that polyols, especially glycerol, are the only organic solutes used to regulate intracellular osmotic pressure (Meikle et al., 1991).

The ability to retain a greater proportion of a constant total (intra- and extracellular) amount of glycerol intracellularly was proposed by Brown (1978) as an essential characteristic of an osmotolerant yeast such as $Z$. rouxii, whereas a non-osmotolerant yeast such as Sacc. cerevisiae produces increasing amounts of glycerol and retains intracellularly a decreasing proportion of the total. The four yeasts $(C$. cacaoi, $C$. magnoliae, $P$. sorbitophila and $Z$. bisporus) were found to have a minimum growth $a_{\mathrm{w}}$ value adjusted with glucose, of less than 0.84 which, according to the taxonomic literature (Barnett et al., 1983), would classify them as osmotolerant yeasts. Their behaviour in producing and accumulating glycerol was, however, more similar to that observed with Sacc. cerevisiae (Brown, 1978) which suggests that the exceptional osmotolerance and different behaviour of $Z$. rouxii may be unique amongst the yeasts. Calculations of the intracellular $a_{\mathrm{w}}$ from the glycerol concentration (Weast, 1984) indicated that in most instances sufficient glycerol was accumulated by the four yeasts to enable the intra- and extracellular $a_{\mathrm{w}}$ to attain an equilibrium. Apparently, these yeasts are osmotolerant by virtue of their ability to produce increasing amounts of glycerol with decreasing $a_{\mathrm{w}}$ rather than to retain a greater proportion of a constant amount of glycerol. Furthermore, the current division of yeasts into osmotolerant and non-osmotolerant has no clear physiological basis.

The increasing ratio (intra-/extracellular concentration) of glycerol with a decrease in $a_{\mathrm{w}}$ indicates that the permeability of the plasma membrane is involved in the water relations of yeasts (Brown, 1978). During osmotic stress, the permeability of the yeast membrane to glycerol and other solutes may be reduced by changes in the phospholipid composition (Watanabe \& Takakuwa, 1984; Tunblad-Johansson \& Adler, 1987) thus allowing solute accumulation. Alternatively, yeasts may maintain high intracellular concentrations by means of active transport of glycerol. Active transport mechanisms regulated by osmotic stress have been described in $D$. hansenii (Adler et al., 1985; Lucas et al., 1990) and $Z$. rouxii (Van Zyl et al., 1990) which enable glycerol to be accumulated intracellularly up to 5000 -fold. Furthermore, the greater retention of glycerol as observed here when the $a_{\mathrm{w}}$ was adjusted with $\mathrm{NaCl}$ than with glucose could be related to the use of a sodium gradient as the driving force to maintain the glycerol gradient.

Financial support from the Foundation for Research Development and the University of the Orange Free State was gratefully received. J. C. du Preez is thanked for reviewing the manuscript.

\section{References}

Adler, L., Blomberg, A. \& Nilsson, A. (1985). Glycerol metabolism and osmoregulation in the salt-tolerant yeast Debaryomyces hansenii. Journal of Bacteriology 162, 300-306.

Barnett, J. A., Payne, R. W. \& Yarrow, D. (1983). Yeasts: Characteristics and Identification. Cambridge \& New York: Cambridge University Press.

Bellinger, Y. \& LARHER, F. (1988). A ${ }^{13} \mathrm{C}$ comparative nuclear magnetic resonance study of organic solute production and excretion by the yeasts Hansenula anomala and Saccharomyces cerevisiae in saline media. Canadian Journal of Microbiology 34, 605-612.

Brown, A. D. (1978). Compatible solutes and extreme water stress in eukaryotic microorganisms. Advances in Microbial Physiology 17, $181-242$.

Brown, A. D., Mackenzie, K. F. \& Singh, K. K. (1986). Selected aspects of microbial osmoregulation. FEMS Microbiology Reviews 39, 31-36.

Chirife, J., Alzamora, S. M. \& Ferro Fontan, C. F. (1983). Microbial growth at reduced water activities: studies of $a_{\mathrm{w}}$ prediction in solutions of compatible solutes. Journal of Applied Bacteriology 54, 339-343.

Chirife, J., Favetro, G. \& Ferro Fontan, C. F. (1984). Microbial growth at reduced water activities: some physicochemical properties of compatible solutes. Journal of Applied Bacteriology 56, 259-268.

CsonkA, L. N. (1989). Physiological and genetic responses of bacteria to osmotic stress. Microbiological Reviews 53, 121-147. 
Edgley, M. \& Brown, A. D. (1978). Response of xerotolerant and non-tolerant yeasts to water stress. Journal of General Microbiology 104, 343-345.

Новот, J. A. \& Jennings, D. H. (1981). Growth of Debaryomyces hansenii and Saccharomyces cerevisiae in relation to $\mathrm{pH}$ and salinity. Experimental Mycology 5, 217-228.

HockING, A. D. \& NorTON, R. S. (1983). Natural-abundance ${ }^{13} \mathrm{C}$ nuclear magnetic resonance studies on the internal solutes of xerophilic fungi. Journal of General Microbiology 129, 2915-2925.

Jermini, M. F. G. \& SCHMIDT-LoRENZ, W. (1987). Growth of osmotolerant yeasts at different water activity values. Journal of Food Protection 50, 404410.

LuCAS, C., Da Costa, M. \& VAN Uden, N. (1990). Osmoregulatory active sodium-glycerol co-transport in the halotolerant yeast Debaryomyces hansenii. Yeast 6, 187-191.

Meikle, A. J., Chudek, J. A., Reed, R. H. \& Gadd, G. M. (1991). Natural abundance ${ }^{13} \mathrm{C}$-nuclear magnetic resonance spectroscopic analysis of acyclic polyol and trehalose accumulation by several yeast species in response to salt stress. FEMS Microbiology Letters 82, 163-168.

NoBre, M. F. \& DA CosTa, M.S. (1985). Factors favouring the accumulation of arabitol in the yeast Debaryomyces hansenii. Canadian Journal of Microbiology 31, 467-471.

Norkrans, B. \& Kylin, A. (1969). Regulation of potassium to sodium ratio and the osmotic potential in relation to salt tolerance in yeasts. Journal of Bacteriology 100, 836-845.

NORRISH, R. S. (1966). An equation for the activity coefficients and equilibrium relative humidities of water in confectionary syrups. Journal of Food Technology 1, 25-39.

Reed, R. H., Chudek, J. A., Foster, R. \& Gadd, G. M. (1987). Osmotic significance of glycerol accumulation in exponentially growing yeasts. Applied and Environmental Microbiology 53, 21192123.

Restaino, L., Bills, S., Tscherneff, K. \& Lenovich, L. M. (1983). Growth characteristics of Zygosaccharomyces rouxii isolated from chocolate syrup. Applied and Environmental Microbiology 45, 1614-1621.

Robinson, R. A. \& Stokes, R. H. (1959). Electrolyte Solutions. New York: Academic Press.

SPENCER, J. F. T. \& SPENCER, D. M. (1978). Production of polyhydroxy alcohols by osmotolerant yeasts. In Economic Microbiology, vol. 2, pp. 393-425. Edited by A. H. Rose. London: Academic Press.

TILBURY, R. H. (1980). Xerotolerant (osmophilic) yeasts. In Biology and Activities of Yeasts, pp. 152-179. Edited by F. A. Skinner, S. M. Passmore \& R. R. Davenport. London: Academic Press.

TOKUOKA, K. \& IsHITANI, T. (1991). Minimum water activities for the growth of yeasts isolated from high-sugar foods. Journal of General and Applied Microbiology 37, 111-119.

TUNBLAD-JohansSON, I. \& ADLER, L. (1987). Effects of sodium chloride concentration on phospholipid fatty acid composition of yeasts differing in osmotolerance. FEMS Microbiology Letters 43, 275-278.

VAN Eck, J. H., Prior, B. A. \& Brandt, E. V. (1989). Accumulation of polyhydroxy alcohols by Hansenula anomala in response to water stress. Journal of General Microbiology 135, 3505-3513.

VAN ZYL, P. J. \& PRIOR, B. A. (1990). Adaptation of Zygosaccharomyces rouxii to changes in water activity in transient continuous culture. Biotechnology Letters 12, 361-366.

Van ZyL, P. J., Killan, S. G. \& Prior, B. A. (1990). The role of an active transport mechanism in glycerol accumulation during osmoregulation by Zygosaccharomyces rouxii. Applied Microbiology and Biotechnology 34, 231-235.

WATANABE, Y. \& TAKAKUWA, M. (1984). Effects of sodium chloride on lipid composition of Saccharomyces rouxii. Agricultural and Biological Chemistry 48, 2415-2422.

WEAST, R. C. (1984). In CRC Handbook of Chemistry and Physics, 65th edition, p. D235 Edited by R. C. Weast. Boca Raton: CRC Press.

WICKeRHAM, L. S. (1951). Taxonomy of yeasts. Technical Bulletin no. 1029. Washington, DC: United States Department of Agriculture.

Yancey, P. H., Clark, M. E., Hand, S. C., Bowlus, R. D. \& Somero, G. N. (1982). Living with water stress: evolution of osmolyte systems. Science 217, 1214-1222. 